\title{
Efficient Determination of the Enantiomeric Purity and Absolute Configuration of Flavanones by Using (S)-3,3'-Dibromo-1,1'-bi-2-naphthol as a Chiral Solvating Agent
}

Guoxin Du, ${ }^{\dagger, \ddagger}$ Yisu Li, ${ }^{\ddagger}, \S$ Shunan Ma, Rui Wang, ${ }^{\dagger}$ Bo Li, , , FujiangGuo, ${ }^{*, \dagger}$ Weiliang Zhu, and Yiming $\mathrm{Li}^{*}, \dagger$

'School of Pharmacy, Shanghai University of Traditional Chinese Medicine, 1200 Cailun Road, Shanghai, 201203, People's Republic of China

${ }^{\ddagger}$ CAS Key Laboratory of Receptor Research, Shanghai Institute of Materia Medica, Chinese Academy of Sciences, 555 Zuchongzhi Road, Shanghai, 201203, People's Republic of China

${ }^{\S}$ Nano Science and Technology Institute, University of Science and Technology of China, 166 Renai Road, Suzhou, 215123, People's Republic of China 
Table of contents

Table S1. ${ }^{1} \mathrm{H}$ NMR Chemical Shift Nonequivalences $\left(\Delta \delta^{\mathrm{rac}}\right)$ of Racemic Bavachinin 7 in the presence of 3 equiv $(S)-\mathbf{1}-\mathbf{6}$

Table S2. $\quad{ }^{1} \mathrm{H}$ NMR Chemical Shift Nonequivalences $\left(\Delta \delta^{\mathrm{rac}}\right)$ of Racemic Bavachinin 7 in the presence of $(S)-1$

Figure S1. Overlaid ${ }^{1} \mathrm{H}$ NMR spectrum $\left(600 \mathrm{MHz}\right.$ in $\mathrm{CDCl}_{3}$ at $\left.298 \mathrm{~K}\right)$ of racemic bavachinin 7 in the presence of $(S)-\mathbf{1}$.

Figure S2. Models for the assignment of the absolute configuration of flavanones from the $\delta^{R}$ and $\delta^{S}$ signs of the H-3a.

Figure S3. Models for the assignment of the absolute configuration of flavanones from the $\Delta \delta^{R S}$ signs of the H-3a.

Figure S4. $\quad{ }^{1} \mathrm{H}$ NMR spectra of 1:3 mixture of natural bavachin 9 and $(S)-\mathbf{1}$.

Figure S5. HPLC-UV-ECD chromatogram of bavachinin 8.

Figure S6. HPLC-UV-ECD chromatogram of bavachin 9.

Figure S7. HPLC-UV-ECD chromatogram of farrerol 10.

Figure S8. HPLC-UV-ECD chromatogram of hesperetin 11.

Figure S9. HPLC-UV-ECD chromatogram of naringenin 12. 
Table S1. ${ }^{1} \mathrm{H}$ NMR Chemical Shift Nonequivalences $\left(\Delta \delta^{\text {rac }}\right)$ of Racemic Bavachinin 7 in the presence of 3 equiv $(S)-1-6$

$\begin{array}{ccc}\text { Entry } & \text { CSA } & \Delta \delta^{\mathrm{rac}}(\mathrm{ppm}) \\ 1 & (S)-\mathbf{1} & 0.012 \\ 2 & (S)-\mathbf{2} & 0.019 \\ 3 & (S)-3 & 0.000 \\ 4 & (S)-\mathbf{4} & 0.006 \\ 5 & (S)-\mathbf{5} & 0.000 \\ 6 & (S)-6 & 0.003\end{array}$


Table S2. ${ }^{1} \mathrm{H}$ NMR Chemical Shift Nonequivalences $\left(\Delta \delta^{\text {rac }}\right)$ of Racemic Bavachinin 7 in the presence of $(S)-1$

$\begin{array}{ccc}\text { Entry } & \text { Equiv of }(S)-\mathbf{1} & \Delta \delta^{\mathrm{rac}}(\mathrm{ppm}) \\ 1 & 1 & 0.005 \\ 2 & 2 & 0.010 \\ 3 & 3 & 0.012 \\ 4 & 6 & 0.028 \\ 5 & 12 & 0.046 \\ 6 & 18 & 0.044\end{array}$




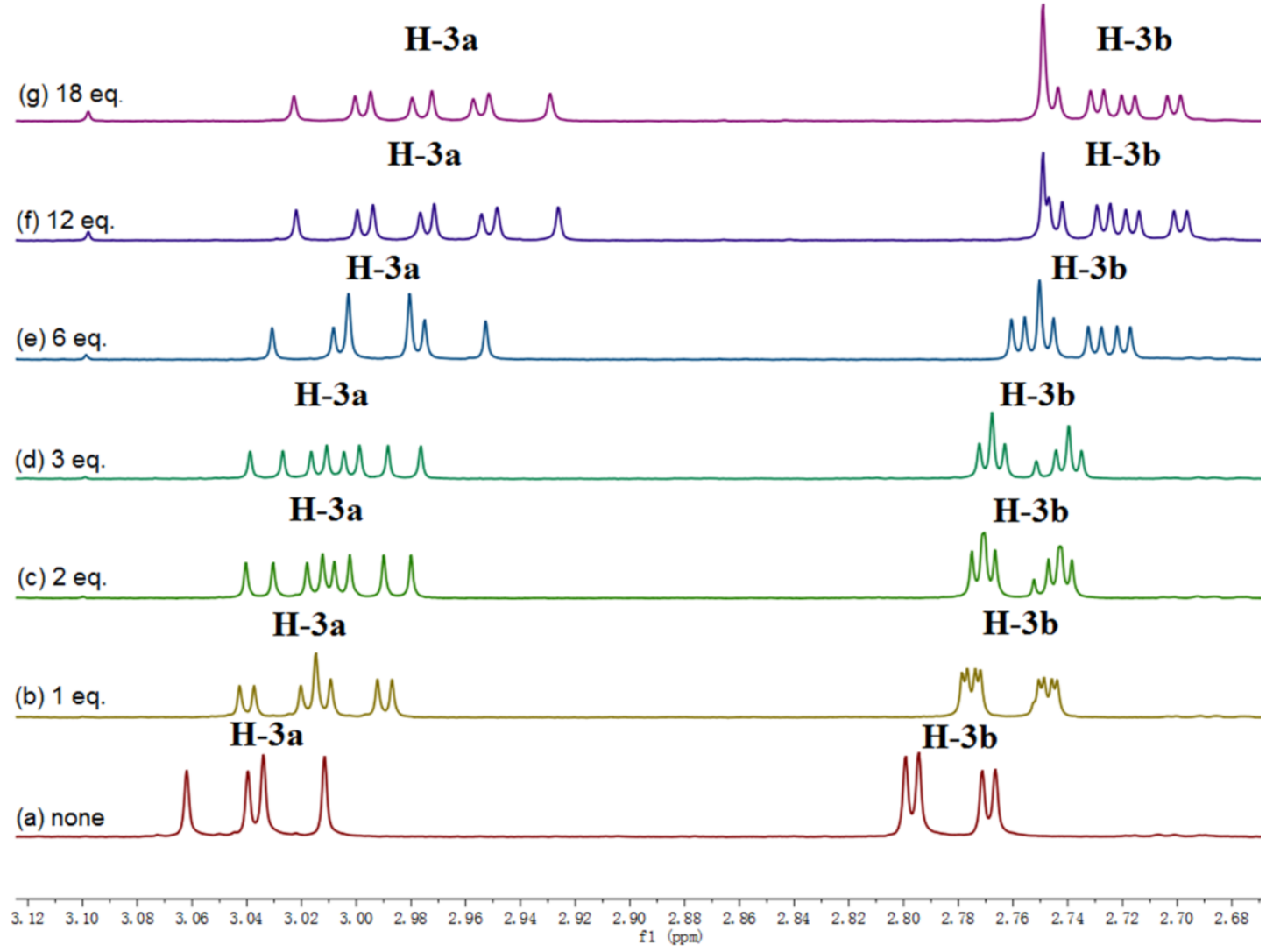

Figure S1. Overlaid ${ }^{1} \mathrm{H}$ NMR spectrum $\left(600 \mathrm{MHz}\right.$ in $\mathrm{CDCl}_{3}$ at $\left.298 \mathrm{~K}\right)$ of racemic bavachinin

$\mathbf{7}$ in the presence of $(S)-\mathbf{1}$. 


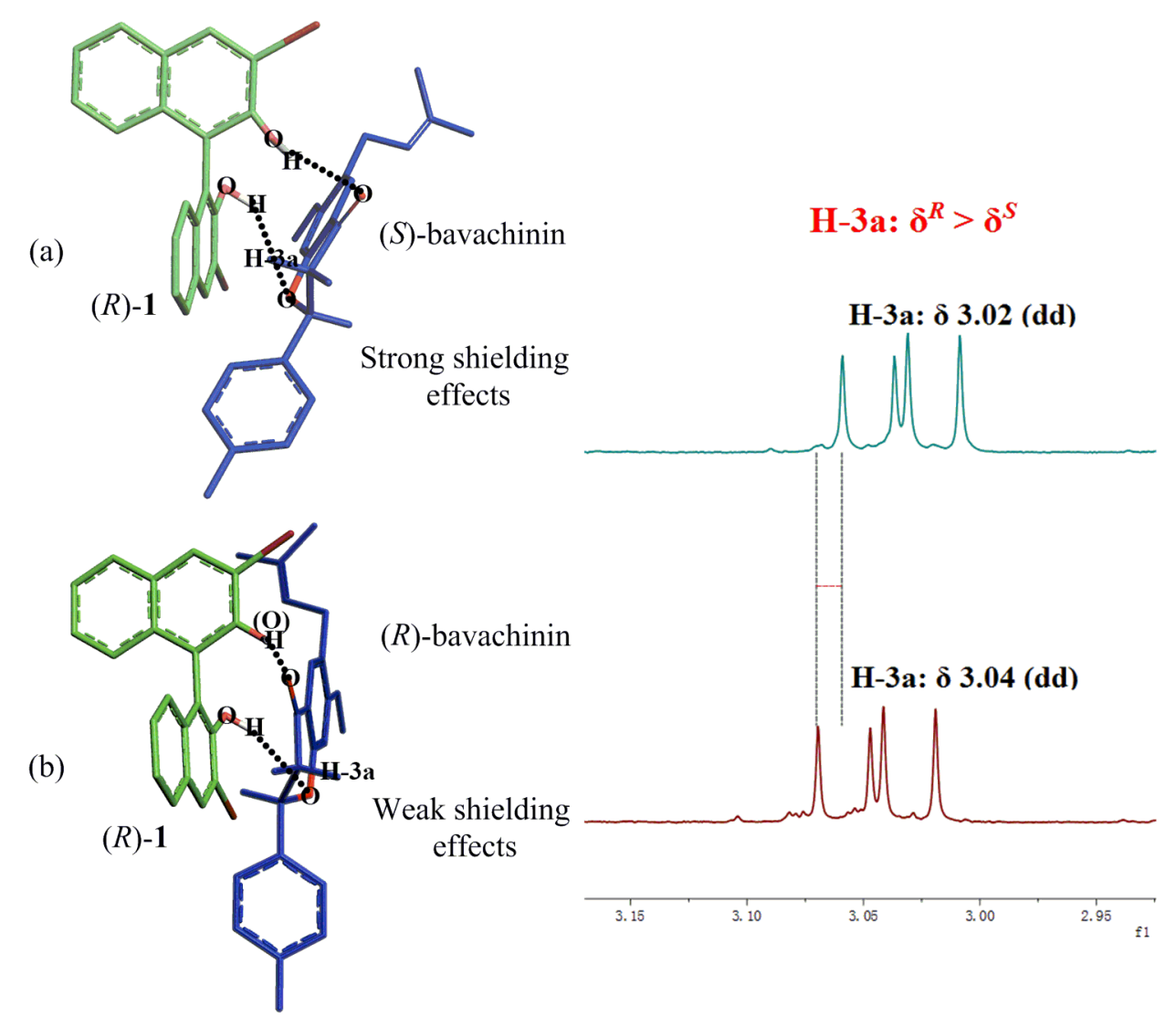

Figure S2. Models for the assignment of the absolute configuration of flavanones from the $\delta^{R}$ and $\delta^{S}$ signs of H-3a: (a) $1: 3$ mixture of $(S)$-bavachinin and $(R)-1$; (b) $(R)$-bavachinin with $(R)$-1. The black dashed line represents a H-bond. 
(a)

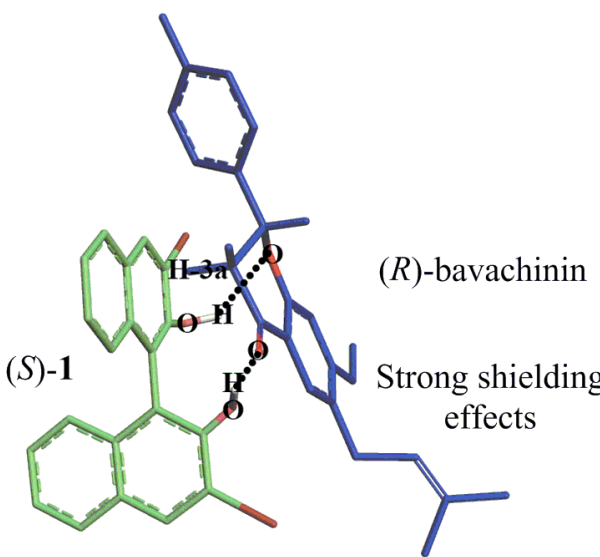

H-3a: $\Delta \boldsymbol{\delta}^{R S}>\mathbf{0}$

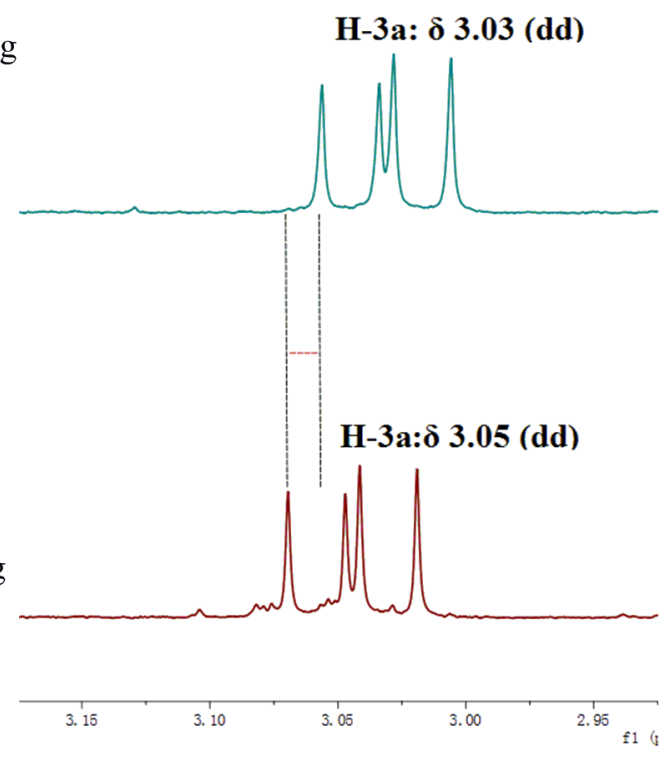

Figure S3. Models for the assignment of the absolute configuration of flavanones from the $\Delta \delta^{R S}$ signs of H-3a: (a) $1: 3$ mixture of $(R)$-bavachinin and $(S)$-1; (b) $(R)$-bavachinin with (R)-1. The black dashed line represents a H-bond. 


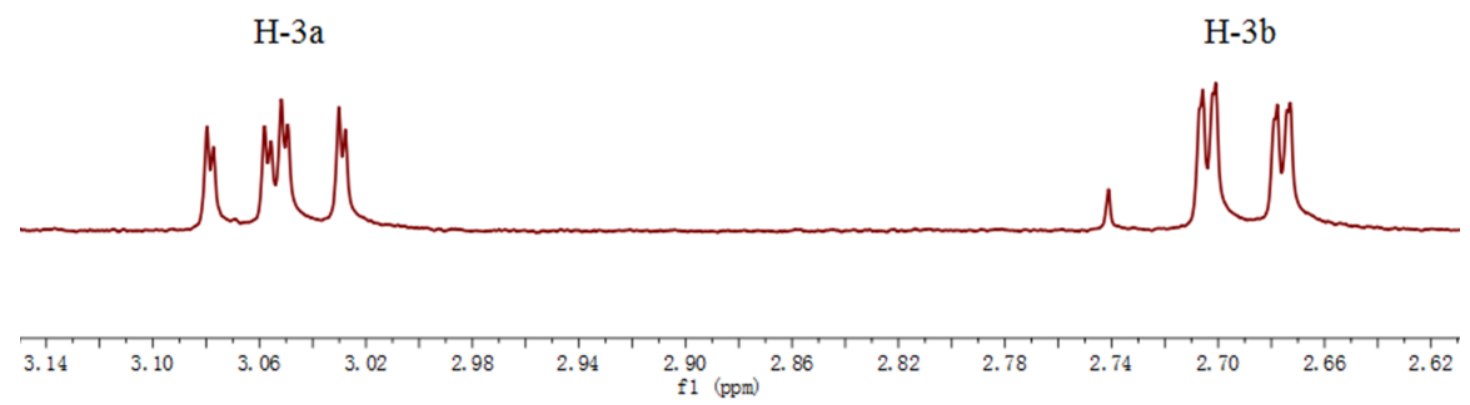

Figure S4. ${ }^{1} \mathrm{H}$ NMR spectra of 1:3 mixture of natural bavachin 9 and $(S)-1(600 \mathrm{MHz}$ in acetonitrile- $d_{3}$ at $\left.298 \mathrm{~K}\right)$. 

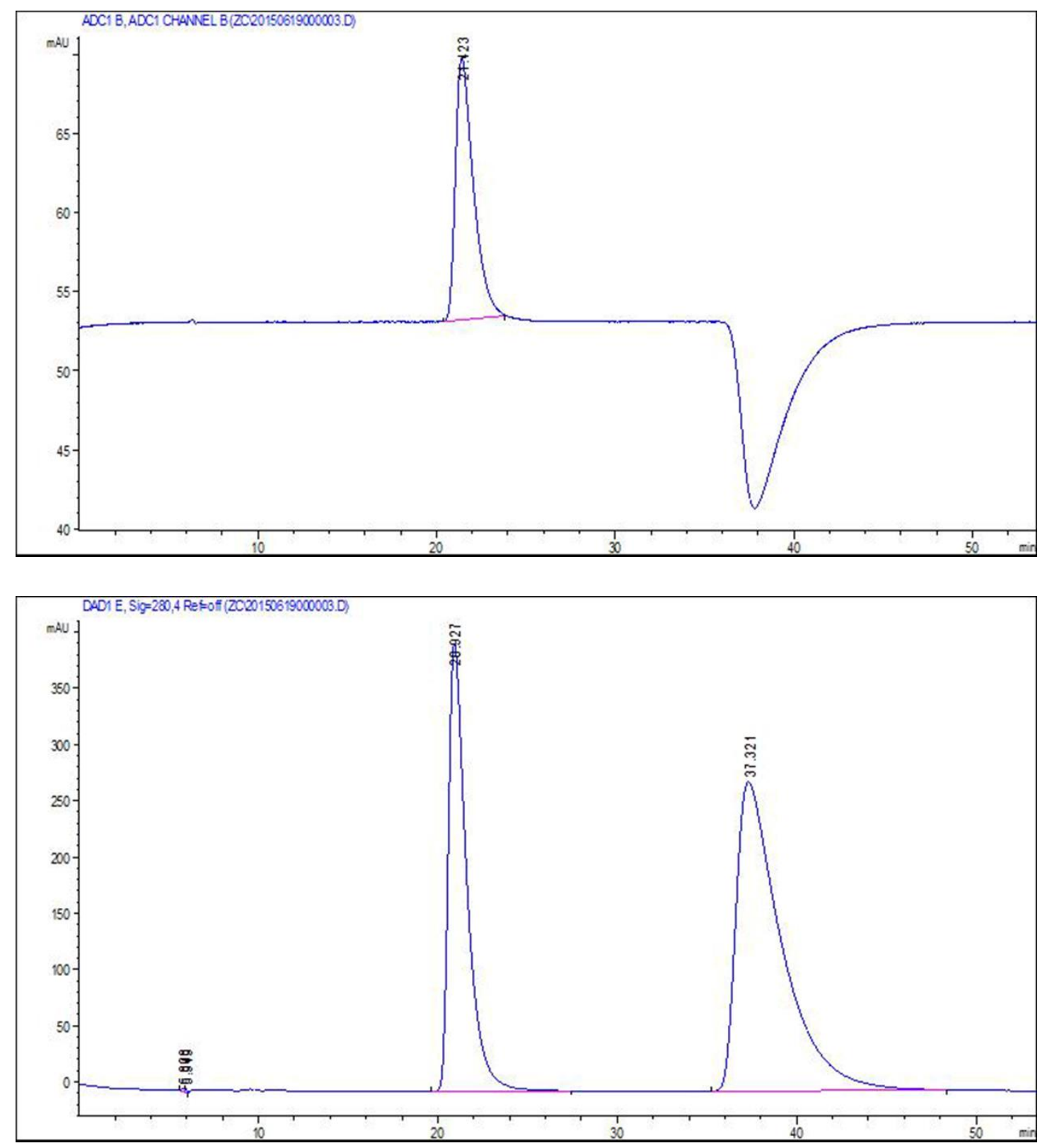

\# 时间 峰面积峰高 峰宽 对称因子 峰面积 \%

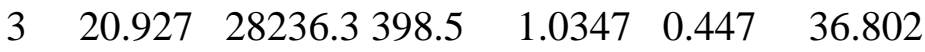

$\begin{array}{lllllll}4 & 37.321 & 48433.3 & 275.2 & 2.3976 & 0.347 & 63.127\end{array}$

Figure S5. HPLC-UV-ECD chromatogram of bavachinin 8 . 

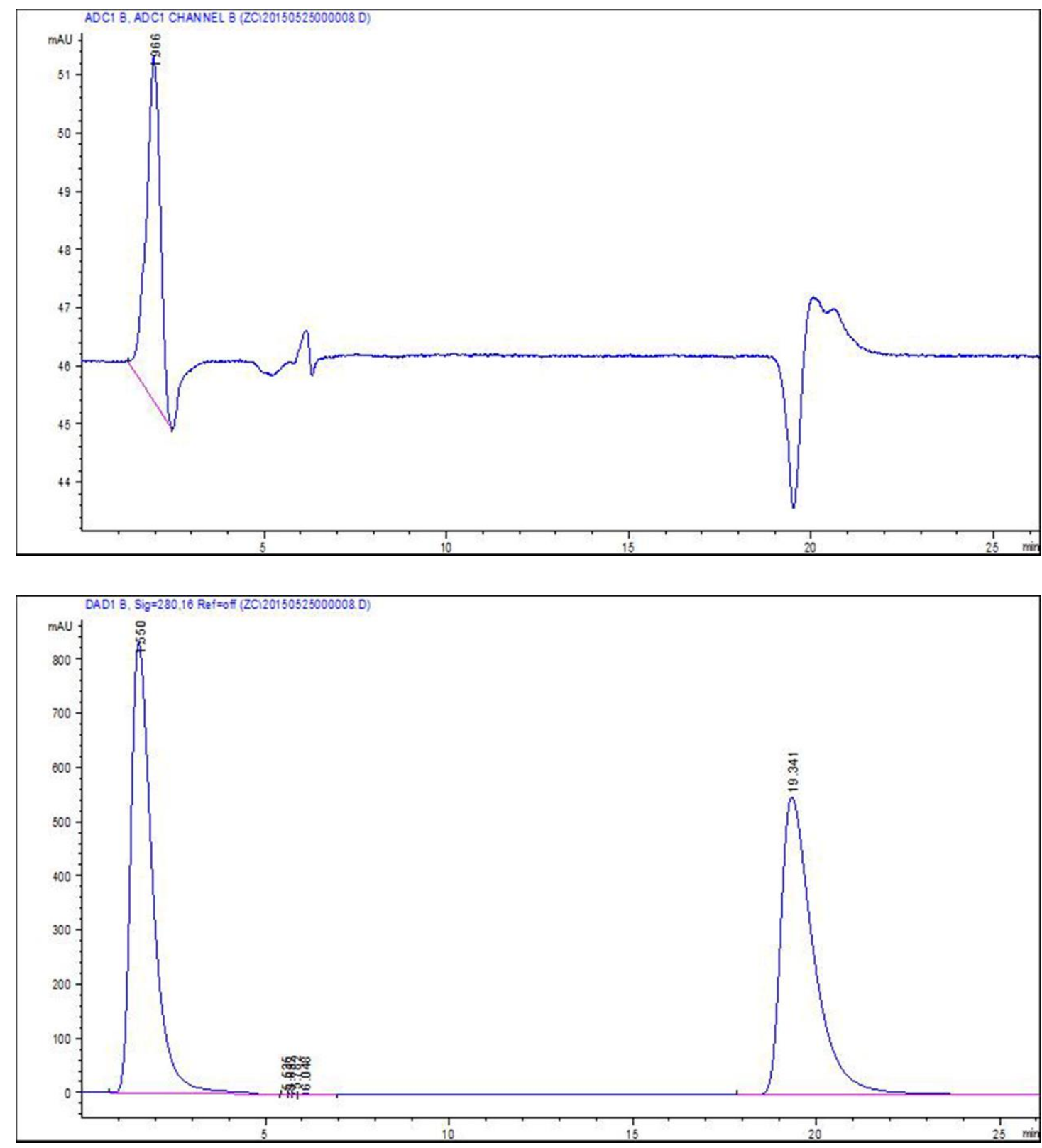

\# 时间 峰面积峰高 峰宽 对称因子 峰面积 \%

$\begin{array}{lllllll}1 & 1.55 & 33944.1 & 831.9 & 0.614 & 0.531 & 50.032\end{array}$

$\begin{array}{lllllll}6 & 19.341 & 33827 & 549.6 & 0.9181 & 0.464 & 49.860\end{array}$

Figure S6. HPLC-UV-ECD chromatogram of bavachin 9. 

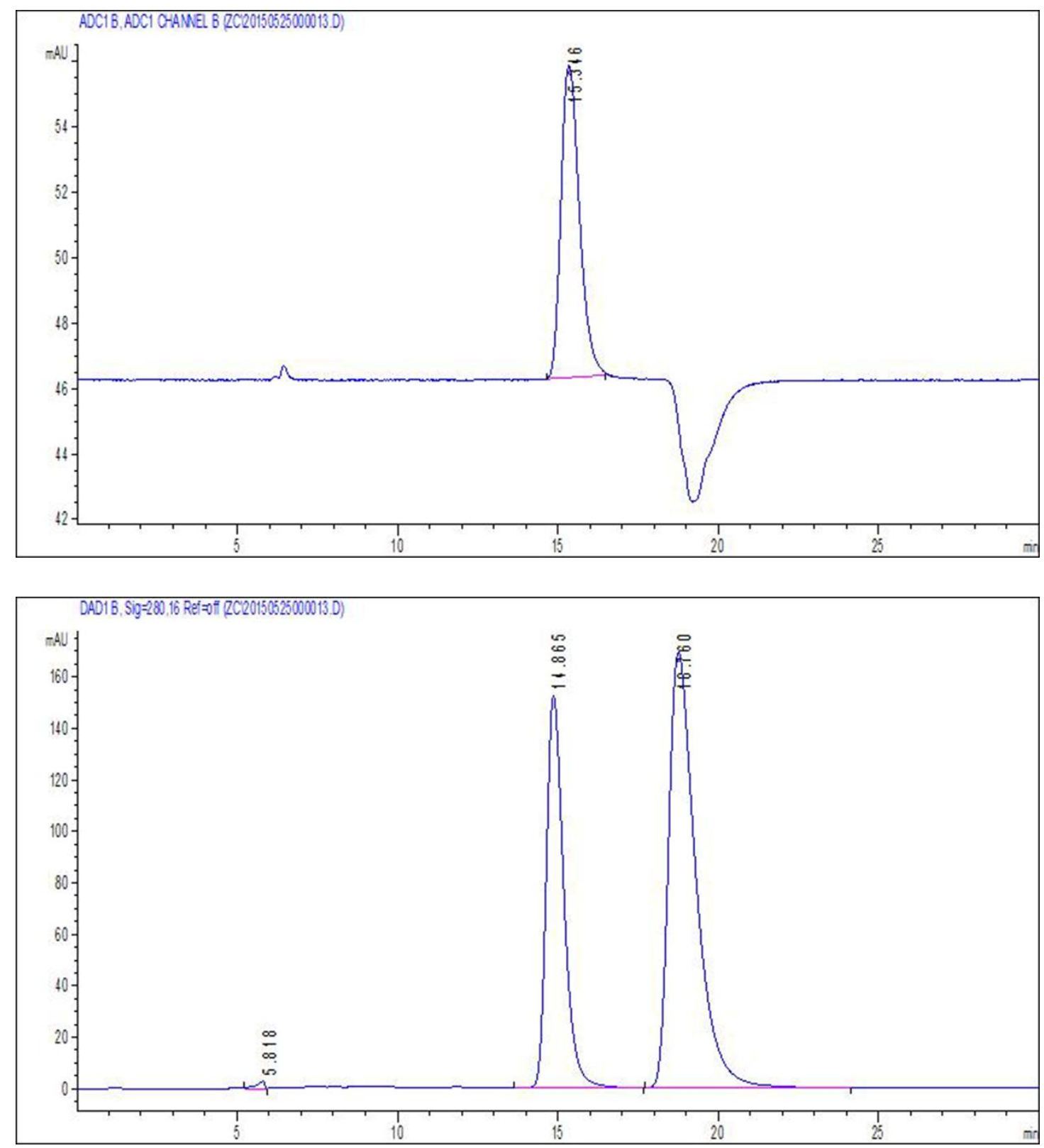

\# 时间 峰面积峰高 峰宽 对称因子 峰面积 \%

$\begin{array}{lllllll}2 & 14.865 & 5760.4 & 152.5 & 0.5788 & 0.656 & 36.448\end{array}$

$\begin{array}{lllllll}3 & 18.76 & 9994.5 & 169.4 & 0.8922 & 0.516 & 63.239\end{array}$

Figure S7. HPLC-UV-ECD chromatogram of farrerol 10. 

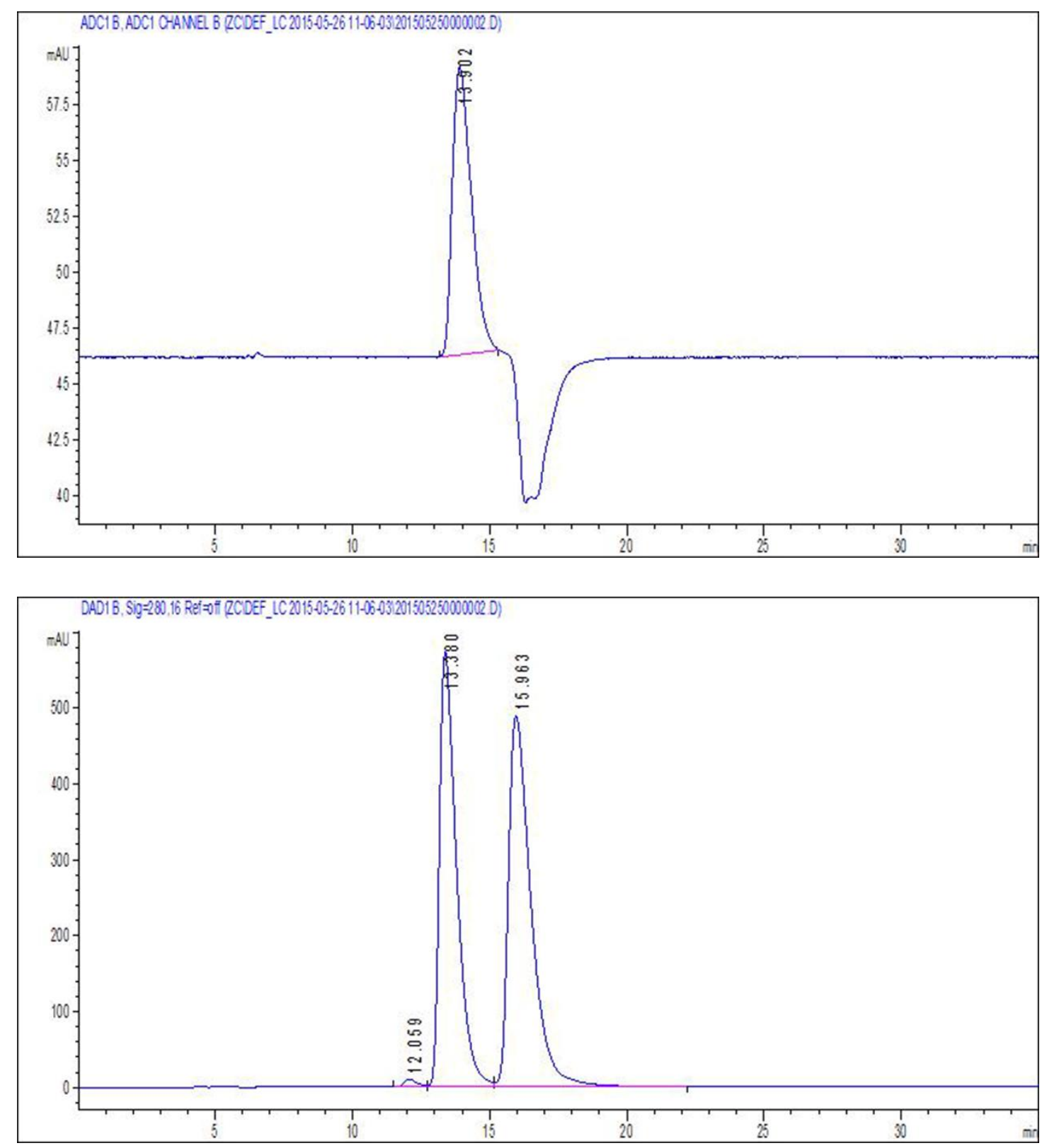

\# 时间 峰面积峰高 峰宽 对称因子 峰面积 \%

$\begin{array}{llllll}2 & 13.38 & 24867.9573 .4 & 0.648 & 0.471 & 46.774\end{array}$

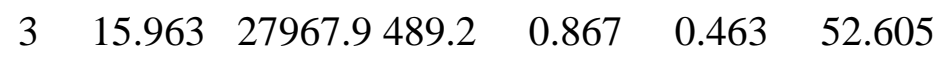

Figure S8. HPLC-UV-ECD chromatogram of hesperetin 11. 

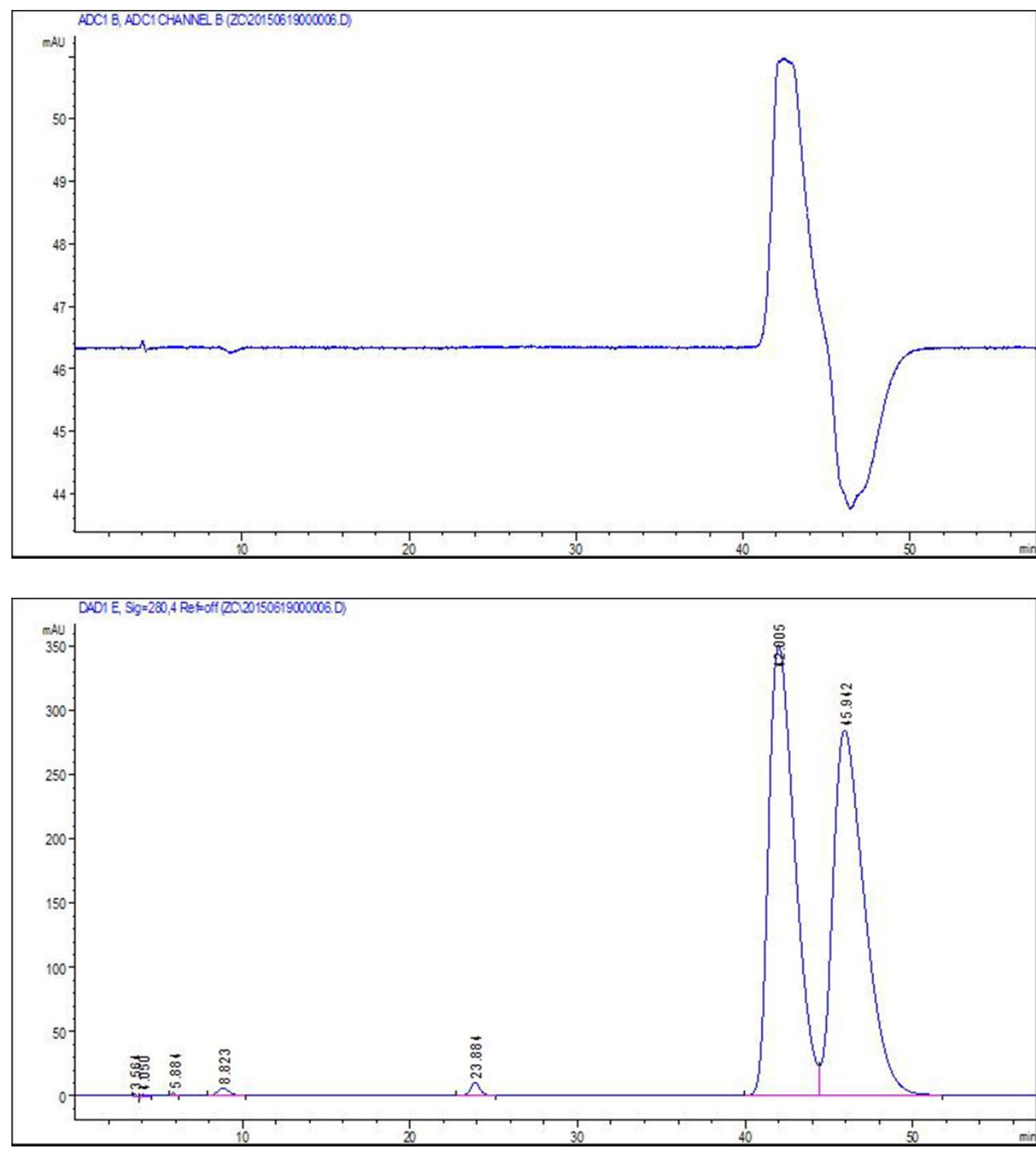

\# 时间 峰面积峰高 峰宽 对称因子 峰面积 \%

$\begin{array}{llllll}6 & 42.005 & 37937.2350 .8 & 1.6198 & 0.589 & 49.981\end{array}$

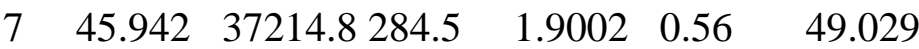

Figure S9. HPLC-UV-ECD chromatogram of naringenin 12. 